\title{
Die ubiquitäre Umweltpräsenz von Kunststoffen: ein Makro- oder Mikroproblem?
}

Kunststoffmaterialien und -produkte sind aus unserem Alltag nicht mehr wegzudenken. Viele Annehmlichkeiten unseres alltäglichen Lebens wären genauso wie viele technologische Entwicklungen in den unterschiedlichsten Sektoren ohne diesen Werkstoff nicht möglich (gewesen). Der nicht wertschätzende, kurzsichtige und unachtsame Umgang mit diesen Materialien hat aber dazu geführt, dass wir Kunststoffe unterschiedlichster Herkunft und deren Partikel mittlerweile in allen Umweltmedien sowie in Lebewesen, bis hin zum Menschen, vorfinden. Meinungen und Medienberichte zu dieser Problematik gibt es viele. Fakten, belastbare Daten und aussagekräftige Studien hingegen sind rar.

Den Siegeszug trat die Polymerchemie zu Beginn des 20. Jahrhunderts an, die großtechnische Produktion etablierte sich in den 1950er-Jahren und Kunststoffe wurden rasch zu bedeutenden Werkstoffen, die viele Bereiche der Gesellschaft und den technischen Fortschritt verändert haben. Im Jahr 2015 wurden weltweit 322 Mio. $t$ Kunststoffe (PlasticsEurope 2017) und in der EU etwa 49 Mio. $t$ (EU COM 2018) produziert. Ihren Erfolg verdanken Kunststoffe, neben dem besonders vorteilhaften Merkmal des geringen Gewichts, v.a. den günstigen Produktionskosten, den vielfältigen Verarbeitungsformen, ihrer Modifizierbarkeit und der Diversität ihrer Eigenschaften, die dazu führten, dass sie in den vergangenen Jahrzehnten in vielen Bereichen traditionelle Werkstoffe sehr schnell ersetzten. Kunststoffe werden z.B. im großen Maßstab im Verpackungsbereich eingesetzt und schützen oder verlängern die Lebensdauer wertvoller Produkte, wie Lebensmittel, Medikamente oder elektronische Geräte. Die Verpackungen selbst haben aber meist nur einen sehr kurzen Lebenszyklus.

Die für den Einsatz und Gebrauch vorteilhaften, vielfältigen Eigenschaften führen am Ende ihrer Nutzungsphase dazu, dass Kunststoffprodukte zu Abfällen mit „besonderen Herausforderungen“ werden. Die Materialvielfalt, Materialverbunde durch Compoundierungen und die Zugabe unterschiedlicher Füllstoffe und umweltrelevanter Additive (wie Flammhemmer, Weichmacher etc.) erschweren ein hochwertiges Materialrecycling. Für den Entsorger oder Verwerter ist in den meisten Fällen nicht mehr nachvollziehbar, welche Zumischungen in den Altkunstoffen enthalten sind und folglich in Sekundärrohstoffe bzw. Recyclate unkontrolliert „verschleppt“ werden können. Diese Additive können auch während des Zerfalls von Kunststoffabfällen in der Umwelt freigesetzt werden und stellen daher bei der Diskussion um die Problematik von Kunststoffpartikeln in der Umwelt einen wesentlichen Aspekt dar.

Um den unkontrollierten Eintrag von Makro- und Mikrokunststoffpartikeln in die Umwelt vermeiden zu können, ist es wichtig, den Ursprung und die „Entstehungsgeschichte“ des Materials zu kennen. Bei Mikrokunststoffpartikeln unterscheidet man diesbezüglich zwischen primärem und sekundärem „Mikroplastik“. Primäres Mikroplastik wird entweder bereits in mikrometrischem Maßstab hergestellt (Typ A), wie beispielsweise Kunststoffpellets oder Kunststoffpartikel in Kosmetika, oder es wird erst während der Nutzungsphase (Typ B) durch diverse Prozesse freigesetzt, wie z. B. Reifenabrieb, welcher als eine der größten Emissionsquellen dieses Typs identifiziert wurde (vgl. z.B. Bertling et al. 2018). Während man die Freisetzung von primärem Mikroplastik vom Typ A durch regulatorische (z.B. Verbot von Mikrokunststoffen in Kosmetika) oder technische Maßnahmen (Reduktion von z. B. Pellet-Verlusten im Produktionsprozess) in Angriff nehmen kann, ist die Reduktion von Mikroplastikemissionen vom Typ B durch Fortschritte in der Materialentwicklung möglich. Weiters unterscheidet man sekundäres Mikroplastik, das durch physikalische, (bio-)chemische und biologische Prozesse in der Umwelt unkontrolliert aus gelitterten Makrokunststoffen (gemäß derzeitigem Verständnis sind das makroskopische Kunststoffteilchen größer $5 \mathrm{~mm}$ ) entsteht. Ein Ansatz, die Umweltproblematik 


\section{Editorial}

von sekundärem Mikroplastik zu entschärfen, ist die Entwicklung von biologisch abbaubaren Kunststoffen. Die Materialsubstitution durch biologisch abbaubare Kunststoffe erscheint aber nur für einige wohlüberlegte Anwendungsbereiche (z. B. bei Produkten mit langjährigem Freilandeinsatz im landund forstwirtschaftlichen Bereich) zielführend. Eine 1:1 Materialsubstitution bei alltäglichen Einwegund Wegwerfprodukten ist kritisch zu betrachten. Rebound-Effekte können eintreten, nämlich dann, wenn die Bevölkerung „mit gutem Gewissen“ diese Kunststoffmaterialien vermehrt in der Natur zurücklässt, da diese ja „biologisch abbaubar“ sind. Vielmehr muss eine Systemsubstitution hin zu Mehrwegprodukten mit längeren Nutzungszyklen stattfinden.

Einen bedeutenden Eintragspfad für Makrokunststoffe in die Umwelt stellen vor allem die unsachgemäße Entsorgung und das achtlose Wegwerfen („Littering“) von Kunststoffprodukten bzw. -abfällen dar (siehe dazu Beitrag von Huter und Pomberger). Kunststoffverpackungen, vorwiegend aus dem Lebensmittel- und Getränkebereich, sind dabei stück- wie auch masse- und volumenmäßig besonders relevant (siehe dazu Beiträge von Hietler und Pladerer sowie Mayerhofer et al.). Global hochgerechnete und mit Unsicherheiten behaftete Zahlen des World Economic Forums zeigen beispielhaft für das Jahr 2013, dass weltweit ca. 32 Masse-\% der produzierten Kunststoffverpackungen unkontrolliert aus dem Wirtschafts- bzw. Abfallwirtschaftssystem verloren gehen und sich in den verschiedenen Umweltmedien anreichern. $40 \%$ der Kunststoffverpackungen werden kontrolliert deponiert und $14 \%$ thermisch behandelt. Nur ca. $2 \%$ werden qualitätsgleich recycelt, $8 \%$ einer kaskadischen Verwertung zugeführt (d.h. aus einer lebensmitteltauglichen Verpackung wird z. B. ein Blumentopf), und $4 \%$ werden als Prozessverlust in der Behandlung definiert (World Economic Forum et al. 2016).

Hauptgründe für diese beträchtlichen globalen „Systemverluste“ sind neben fehlender bzw. fehlgeleiteter Abfallwirtschaft in vielen Entwicklungs- und Schwellenländern auch der unachtsame Umgang mit Kunststoffmaterialien generell. In unsere Gesellschaft wird der Wert von Kunststoffmaterialien meist wesentlich weniger hoch geschätzt als bei anderen Materialien, weil Kunststoffe bzw. die Produkte daraus häufig preiswerter sind als ihre Pendants aus Holz, Glas oder Metall, und Kunststoffe zudem häufig für Einwegprodukte verwendet werden, die sich durch geringere Haltbarkeit und kurze Nutzungsphasen auszeichnen (UBA 2019). Kunststoffe sind aber durchaus komplexe und mit entsprechend hohem Energieeinsatz produzierte Werkstoffe. Die zugrundeliegenden Ressourcen gehen verloren, wenn Kunststoffabfälle gelittert werden, diese in der Umwelt zu kleineren Partikeln zersetzt und dissipativ verteilt werden. Es benötigt einen entsprechend hohen Energieeinsatz, diese Partikel aus den unterschiedlichen Umweltkompartimenten für eine mögliche Kreislaufführung rückzugewinnen oder zum Schutz der Natur zu entfernen.

Die Auswirkungen durch Makro- wie Mikrokunststoffe in unserer Umwelt sind vielfältig. Einige Effekte sind klar ersichtlich, wie die (landschafts-ästhetische) Beeinträchtigung durch die Makro-Vermüllung der Strände und Meere, Flussufer und Flüsse, oder die Verhedderung und Verletzung von Wasserund Landtieren, während störende Einflüsse auf das Edaphon, der im Boden lebenden Organismen, oder Veränderungen der Bodeneigenschaften durch Mikrokunststoffteilchen auf den ersten Blick nicht so leicht erkennbar sind. Die möglichen Gefährdungen unserer Ökosysteme und ihrer Biota, inklusive uns Menschen, durch Mikrokunststoffe sind bis dato erst ansatzweise erforscht (siehe dazu Beitrag von Fürhacker). Relativ neu ist die Erkenntnis, dass sich an den Mikrokunststoffpartikeln sowohl organische wie metallische Schadstoffe, die sich schon in der Umwelt befinden, anreichern können (vgl. Hildebrandt et al. 2020). Zur Frage welche und wie viele umwelttoxikologisch relevante Stoffe und Metalle in und an den Kunststoffpartikeln akkumulieren, gibt es bislang kaum verlässliche wissenschaftliche Untersuchungen. Wenn aus „Mikro“ in der Umwelt in weiterer Folge sogar noch „Nano“ wird, kommen noch weitere zu berücksichtigende Aspekte und neue Wirkmechanismen hinzu, wie z.B. das Vermögen dieser Nano-Kunststoffteilchen Zellmembrane zu passieren und dabei „Schleusen“ für die anhaftenden Schadstoffe oder Additive zu schaffen (siehe Beitrag Fürhacker). Man könnte abstrahiert und vereinfacht zusammenfassen: Je kleiner die Kunststoffpartikel, desto relevanter aber schwerer einschätzbar ist das potenzielle Umweltproblem. 
Um den unterschiedlichen Wirkmechanismen auf die Spur zu kommen, bedarf es auch neuer analytischer Ansätze und wissenschaftlicher Methoden. Selbst für die „einfache“ Quantifizierung und Detektion von Makro-, Mikro- und Nanokunststoffteilchen in den unterschiedlichen Umweltproben gilt es nach wie vor, verlässliche und vergleichbare sowie praktikable Methoden zu entwickeln und Methodenkombinationen zu optimieren (siehe Beiträge von Spacek et al., Liedermann et al. und Zafiu et al.). Die großen Streubreiten der bisher publizierten Daten zu Makro- und Mikroplastik in Flüssen und Seen sowie im marinen Milieu und in Böden sind nicht nur auf geografische und strukturelle Unterschiede bzw. diverse Bewirtschaftungsformen, sondern auch auf die unterschiedlichen methodischen Vorgehensweisen und spezifischen Unzulänglichkeiten der eingesetzten Probenahme-, Aufbereitungs- und Analysemethoden zurückzuführen.

Kunststoffverunreinigungen an Stränden und im Meer sind seit den 1970er-Jahren bekannt und dokumentiert. Die größte und wohl „,berühmteste“ Akkumulation von (vorwiegend) Kunststoffabfällen in den Weltmeeren, the Great Pacific Garbage Patch, wird bereits seit Mitte der 1980er-Jahre zunehmend detaillierter beforscht und beschrieben. Das Problem ist seit mehr als 35 Jahren bekannt, dennoch haben wir es bisher nicht geschafft, schlagkräftige Maßnahmen zur Reduktion dieser marinen Deponien umzusetzen. Die Europäische Union versucht nun mit der im Rahmen des Kreislaufwirtschaftspakets eingesetzten „Single-Use Plastic“-Directive, einer Anti-Littering-Richtlinie zur Reduktion der Kunststoffabfälle in den Ozeanen, dieser Entwicklung entgegenzuwirken. Das ist ein wichtiges umwelt- und gesellschaftspolitisches Signal, ob die darin vorgegebenen Maßnahmen die erwartete breite Wirkung zeigen werden, gilt es abzuwarten. Dies vor allem auch vor dem Hintergrund, dass der hauptsächliche Eintrag von Kunststoffen ins Meer aus Küstenstaaten stattfindet, die nicht zur EU gehören (Jambeck et al. 2015), wie China, Indien und Südasien sowie Länder in Afrika und dem Nahen Osten (Boucher und Friot 2017). Betrachtet man rein den jährlichen Kunststoffeintrag über Flüsse, so werden laut den Hochrechnungen von Schmidt et al. (2017) jahreszeitenabhängig zwischen 88 bis $95 \%$ davon allein von den 10 größten Flusssystemen aus Asien und Afrika ins Meer transportiert. Als größter Verursacher wird hierbei der Jangtse-Fluss gesehen.

Bei den Modellierungen des Kunststoffeintrags vom Land in die Meere spielt der Status der Entwicklung der Abfallwirtschaft in den jeweiligen Ländern genauso wie die Bevölkerungsdichte und der Urbanisierungsgrad der Küstenregionen eine entscheidende Rolle. Generell gilt dies auch für Eintragsbetrachtungen im terrestrischen Bereich, wobei hier noch weitere Faktoren wie z.B. die Bewirtschaftungsweise der Böden (Düngung, Zugabe von Bodenverbesserungsmitteln, Klärschlämmen und Komposten, Einsatz von Agrarfolien etc.) zu berücksichtigen ist. Die Abfallwirtschaft ist hierbei vor allem hinsichtlich der Bereitstellung von hochwertigen, entsprechend schad- und störstofffreien Komposten gefordert (siehe Beitrag von Zafiu et al.).

Solange Bevölkerungszuwachs und Wirtschaftswachstum stattfinden und folglich damit ein Anstieg des Pro-Kopf-Konsums bzw. des Pro-Kopf-Abfallaufkommens (von Kunststoffprodukten) einhergeht, kann bei gleichbleibenden rechtlichen, wirtschaftlichen und institutionellen Rahmenbedingungen auch mit einem Anstieg des Eintrags von Kunststoffen in unsere Umwelt gerechnet werden. Dies trifft in erster Linie auch auf Entwicklungs- und Schwellenländer zu.

Entsprechendes gesamtheitliches Umdenken und nachhaltige Veränderungen sind daher gefragt, um zielführende Maßnahmen zu setzen, global und auf allen Ebenen, vom Konsumenten über die Produzenten bis hin zu den politischen Entscheidungsträgern - und dies nicht nur in einem Mikroansatz, sondern im Makromaßstab! 


\section{Editorial}

\section{Literatur}

Bertling, J., Bertling, R., und Hamann, L. (2018): Kunststoffe in der Umwelt: Mikro- und Makroplastik. Ursachen, Mengen, Umweltschicksale, Wirkungen, Lösungsansätze, Empfehlungen. Kurzfassung der Konsortialstudie. Oberhausen: Frauenhofer-Institut für Umwelt-, Sicherheits- und Energietechnik UMSICHT.

Boucher, J., Friot, D. (2017). Primary Microplastics in the Oceans. Hrsg.: IUCN. 2017, Gland, Schweiz, ISBN 978-2-83171827-9.

EU COM (European Commission) (2018): Circular Economy Package-Implementation of the Circular Economy Action Plan. Available at: http://ec.europa.eu/environment/circular-economy/index_en.htm. Zugegriffen: 16. März 2019

Hildebrandt L., von der Au M., Zimmermann T., Reese A., Ludwig J., Pröfrock D. (2020): A metrologically traceable protocol for the quantification of trace metals in different types of microplastic, PLoS ONE 15(7): e0236120.

Jambeck, J. R., Geyer, R., Wilcox, C., Siegler, T.R., Perryman, M., Andrady, A., Narayan, R., Law, K.L. (2015): Plastic waste inputs from land into the ocean. Science 347, 768-771.

PlasticsEurope (2017): Plastics - the facts: An analysis of European plastics production, demand and waste data. Available at: https://www.plasticseurope.org/en/resources/publications/274-plastics-facts-2017. Zugegriffen: 13. September 2020

Schmidt C., Krauth T., Wagner S. (2017): Export of Plastic Debris by Rivers into the Sea, Environ. Sci. Technol. 51, 21, 12246-12253.

UBA (2019): Kunststoffe in der Umwelt, Umweltbundesamt Deutschland, Dessau-Roßlau, April 2019, ISSN 2363-832X.

World Economic Forum, Ellen MacArthur Foundation, McKinsey \& Company (2016): The New Plastics Economy - Rethinking the future of plastics (https://www.ellenmacarthurfoundation.org/publications). Zugegriffen: 13. September 2020

\section{Univ.-Prof. DI Dr. M. Huber-}

\section{Humer $(\bowtie)$}

Institut für Abfallwirtschaft, Universität

für Bodenkultur Wien,

Muthgase 107,

1190 Wien, Österreich

marion.huber-humer@boku.ac.at 